\title{
Integrating Provenance Data from Distributed Workflow Systems with ProvManager*
}

\author{
Anderson Marinho ${ }^{1}$, Leonardo Murta ${ }^{2}$, Cláudia Werner' ${ }^{1}$, Vanessa Braganholo ${ }^{1}$, \\ Eduardo Ogasawara ${ }^{1}$, Sérgio Manuel Serra da Cruz ${ }^{1}$, and Marta Mattoso ${ }^{1}$ \\ ${ }^{1}$ Federal University of Rio de Janeiro \\ ${ }^{2}$ Fluminense Federal University \\ \{andymarinho, werner, ogasawara, serra, marta\} @cos.ufrj.br, \\ leomurta@ic.uff.br, braganholo@dcc.ufrj.br
}

\begin{abstract}
Running scientific workflows in distributed environments is motivating the definition of provenance gathering approaches that are loosely coupled to the workflow execution engine. This kind of approach is interesting because it allows both storage and access to provenance data in an integrated way, even in an environment where different workflow systems work together. Therefore, we have proposed a provenance gathering strategy that is independent from the workflow system technology. This strategy has evolved into a provenance management system named ProvManager. In this paper we show how provenance data is captured along in a distributed execution environment with ProvManager and we show its web interface, in which scientists can register experiments, monitor workflow execution, and query provenance data.
\end{abstract}

Keywords: provenance, scientific workflows, distributed environment.

\section{Introduction}

Provenance provides historical information about data manipulated in a workflow [1]. This historical information tells us how data products were generated, showing their transformation processes from primary input and intermediary data. The management of provenance information provides to the scientists a variety of data analyses, such as data quality, audit trails, and experiment documentation [2]. Provenance gathering becomes more complex when the workflow is executed among distributed and heterogeneous execution environments, such as clusters, P2P, grids and clouds.

One can foresee several scenarios of workflow execution in a distributed environment [3]. Each one has its own characteristics that contribute to the complexity of provenance management. In this paper we focus in a scenario where pre-existing workflows were conceived independently, using different scientific workflow management systems (SWfMS). However, these independent workflows needed to be integrated into a complex experiment, which entail some additional manual activities that link such workflows. In this scenario, each SWfMS may manage provenance information in a decentralized and isolated way, meaning that each system considers

* This work was partially sponsored by CNPq and CAPES. 
provenance in a specific granularity, stores the information on a specific language, or even worse, some SWfMS may not even provide a provenance solution at all.

Therefore, a solution to this heterogeneity is to transfer the responsibility of provenance management to an independent provenance system. This system would be responsible for capturing, modeling, storing, and providing queries to an integrated provenance management system of an experiment. The main difficulty of the SWfMS agnostic strategy is that the SWfMS and the provenance management system need to communicate to exchange information. In order to make this communication possible, some solutions $[4,5]$ propose a series of manual activity adaptations over the workflow specification. However, this solution introduces additional overhead to scientists. Some workflow activities used by scientists are third-party codes, which make their adaptation more complex. In many cases, these activities cannot be altered, but only wrapped by other activities.

For that reason, in our previous work [3] we have proposed a strategy for gathering provenance information in a distributed environment. This strategy is independent of workflow system technology and tries to address some problems discussed here. This strategy has evolved into a provenance management system named ProvManager. In addition to the gathering mechanism, ProvManager provides means for modeling, storing, and querying an integrated provenance repository.

\section{ProvManager}

The main focus of ProvManager system is to manage provenance in distributed environments. The main idea is to work as a central repository that stores all the provenance data generated from an experiment. The provenance data are collected by automatically adapting the workflow. Workflow activities are thus configured to send this information via a web services API during the workflow execution.

Figure 1.a illustrates the experiment structure that we use as an example for describing the ProvManager's functionalities. This experiment is segmented in two workflows: one workflow is instantiated in VisTrails, and the other in Kepler. Fig. 1.b shows a fragment of the workflow in VisTrails with more details. The fragment is composed by three activities: GetData, Validate, and Simulate, running on a remote host with IP address 192.168.0.5. In order to capture provenance data from this workflow, the scientist has to publish it in ProvManager, uploading the workflow specification (in the VisTrails case, a .VT file). At this moment, ProvManager configures the workflow, automatically adding special activities that will be responsible for capturing and publishing provenance data in ProvManager (this process is described in [3]) during workflow execution. This process of adding provenance components in the workflow is called instrumentation. At present moment, ProvManager can instrument only workflows executed in Kepler and VisTrails. However, ProvManager works with the concept of plugin to be able to support future extensions of other SWfMS instrumentation mechanisms. Finally, at the end of the instrumentation, a new .VT file is returned to the scientist to be reloaded in VisTrails. During both the instrumentation and execution of the workflow, ProvManager captures provenance data from the workflow and publishes this data in the repository. This repository is a Prolog database, so provenance data are mapped into Prolog predicates. Fig. 1.c shows the .VT file mapped into prolog predicates. 

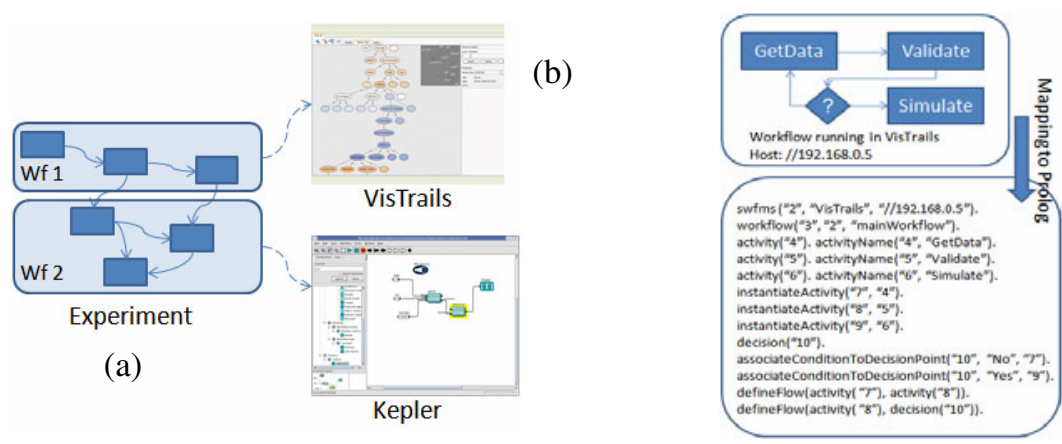

Fig. 1. Experiment example (a), and VisTrails workflow (b) mapped to Prolog predicates (c)

With all the provenance data collected from the experiment, ProvManager makes the experiment analysis process simpler to the scientist since it works as an integrated place for accessing the provenance data, avoiding scientist to visit individually each system responsible for gathering provenance (in our example, Kepler and VisTrails) in a distributed execution environment. Besides, ProvManager provides functionalities to help the scientist manipulate the experiment provenance data, such as high-level provenance query interface, and workflow execution monitoring (Fig. 2).
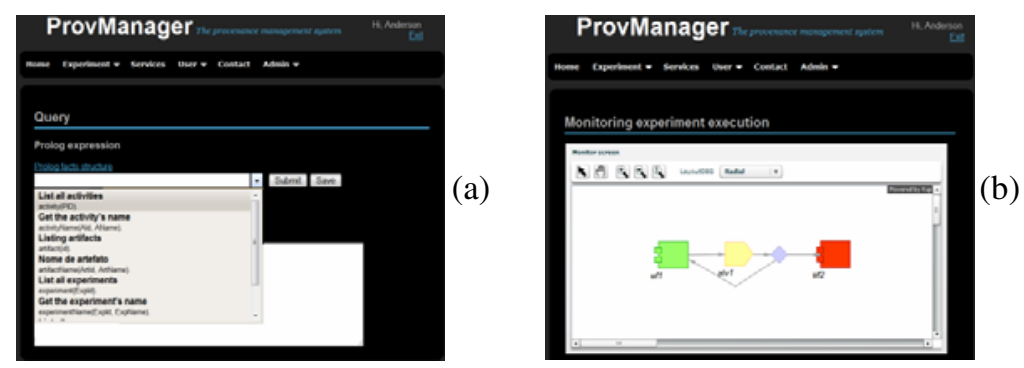

Fig. 2. ProvManager's screens: (a) Query interface; (b) Execution monitoring

\section{References}

[1] Freire, J., Koop, D., et al.: Provenance for Computational Tasks: A Survey. Computing in Science and Engineering 10(3), 11-21 (2008)

[2] Simmhan, Y.L., Plale, B., Gannon, D.: A survey of data provenance in e-science. ACM SIGMOD Record 34(3), 31-36 (2005)

[3] Marinho, A., Murta, L., et al.: A Strategy for Provenance Gathering in Distributed Scientific Workflows. In: IEEE International Workshop on Scientific Workflows, Los Angeles, California, United States (2009)

[4] Simmhan, Y., Plale, B., Gannon, D.: A Framework for Collecting Provenance in DataCentric Scientific Workflows. In: ICWS, pp. 427-436 (2006)

[5] Groth, P., Jiang, S., et al.: An Architecture for Provenance Systems (2006), http: / / eprints.ecs.soton.ac.uk/13216/ (Visited in: July 19, 2010) 